VI.

Aus der Königsberger chirurgischen Klinik des Prof. Dr. Erich Lexer.

\title{
Über Cava-Resektion in einem Fall von Mischgeschwulst der Nierenkapsel.
}

Von

Dr. M. Draudt, Assistenzarzt.

(Mit 2 Abbildungen.)

Die Frage nach der Entstehung der sogenamten embryonalen Mischtumoren der Niere ist noch immer weiterer Klärung und eingehender embryologischer Studien bedürftig. Wenn auch die anf entwicklungsgeschichtlicher Grundlage beruhende Hypothese von Wilms, die die mesodermalen Zellen der Urnierengegend für die Entstehung derartiger Geschwülste verantwortlich macht, die Frage nach der Herkunft aller der verschiedenartigen Bestandteile in gewisser Hinsicht weiter gebracht hat, so hielt doch Wilms selbst die ganze Frage damals noch nicht für spruchreif und hat viele Gegner seiner Anschaungsweise gefunden. Auch gegenwärtig steht man noch nicht auf dem Boden einer einheitlichen Auffassung bezüglich der kindlichen Nierentumoren. Fast jeder Autor hat seine eigne Ansicht, mit der er sich mehr oder weniger einem der großen Heerlager, ob aus der Unnierenanlage oder der fertigen Niere entstanden, anschließt.

Ohne mich zunächst tiefer mit der Streitfrage nach der Geschwulstherkunft zu befassen, möchte ich im folgenden einen höchst bemerkenswerten Fall veröffentlichen, in der Annahme, daß jede derartige Mitteilung, insbesondere, wenn sie gegenüber der Mehrzahl der Beobachtungen Abweichendes enthält, nach mancherlei Seiten hin Interesse bieten kann. Der Fall kam in der Königsberger Klinik von Herrn Prof. Lexer zur Beobachtung, dem ich für freundliche Überlassung zu Dank verpflichtet bin. 
Hermann D., 2 Jahre alt. Etwa seit einem Monat vor der Aufnahme zunehmende Auftreibung des Leibes. Blat soll im Urin niemals vorhanden gewesen sein.

Ziemlich kleines, sehr blasses Kind in mäBigem Ernährungszustand. Ödeme fehlen. Das Abdomen ist fast kugelförmig vorgewölbt durch einen riesigen Tumor, der die ganze rechte Bauchbälfte einnimmt und weit über die Mittellinie nach links hinüberreicht. Oben geht der Tumor direkt in die Leberdámpfung über, nach links reicht er bis zum äuBeren linken Rektusrand, nach unten bis fast zur Symphyse. Die gut verschiebliche Bauchhaut zeigt stark erweiterte Venen. Der Tumor selbst ist auf der Unterlage kaum verschieblich und bewegt sich bei der Atmung nicht mit. Die Oberfläche ist glatt, die Konsistenz hart, ergibt den Eindruck undeutlicher Fluktuation. Im Urin sind Zucker und Eiweiß nicht vorhanden, im reichlichen Sediment keine Tumorbestandteile, kein Blut, reichlich Phosphate.

27. XI. Operation. Prof. Lexer. In Chloroformnarkose, die obne Störung verlief, $20 \mathrm{~cm}$ langer schräger Lumbalschnitt. Nach Durchtrennung von Muskulatur und Fascien muB sogleich das Peritoneum eröffnet werden, das mit dem Tumor in den vorderen Abschnitten fest verwachsen ist und sich von ihm nicht abschieben läßt. Während der Tumor unter schrittweisem Vordringen und Zurückhalten der Därme zu lösen versucht wird, reißt die sehr morsche Masse ein, und es ergießt sich reichlich hämorrhagisch braune Flüssigkeit. Beim weiteren Vordringen nach vorne kommt man in ein eigenartig sulzig-hämorrhagisches Gewebe, das dem Tumor in groBer Ausdehnung dicht anliegt und offenbar einer Blutung entspricht, die vor einigen Tagen stattgefunden zu haben scheint. Nach dem oberen Pol zu liegt der Tumor der Leber an, mit der er aber nicht verwachsen ist. Nach dem nun möglichen Herauswàlzen des Tumors sieht man dicht neben der Wirbelsäule ein gut $3 \mathrm{~cm}$ breites blaues, bandartig zusammengedrücktes Gefäß mit dem Tumor untrennbar zusammenhängen, offenbar die Vena cava!

Da dieselbe von dem Tumor nicht zu lösen ist, wird nun mittels doppelter Umstechung ein etwa $3 \mathrm{~cm}$ langes Stück der Vene reseziert und der nun nur noch mit der Hinterwand festhängende Tumor nach Unterbindung einiger gröBerer Gefäße rasch herausgewalzt. An Stelle des Tumors wird sterile Gaze gebracht, etwa 1 Liter warme Kochsalzlösung in die Bauchhöhle gegossen und die große Wunde bis auf die Tamponöffnung durch einige durchgreifende Nähte geschlossen.

Pat. überstand die Operation, war aber in den nächsten Tagen ganz auBerordentlich blaß. Es war ein merkwürdiger Anblick, beim Verbandwechsel die große, absolut blutleere Wundhölle zu sehen, aus der auch beim Herausnehmen des Tampons kein Blutstropfen sichtbar wurde. Unter öfterem Eingießen von steriler Kochsalzlösung in die Bauchhöhle (die Nähte hatten sehr bald durchgeschnitten und die ganze Wunde lag offen da) erholte sich Pat. immer mehr. Nach etwa 8 Tagen begann eine zunähst minimale Granulationsbildung. Nach einem Vierteljahr war die ganze große Wunde vollkommen geschlossen, so daß Pat. im Ärzteverein rezidivfrei vorgestellt werden konnte. Bemerkenswert ist, daß von Anfang an niemals irgendwelche Stauungserscheinungen in den unteren Extremitäten 
aufgetreten sind. Dagegen hatte sich in ganz hervorragend deutlicher Weise ein Bauchwand-Venennetz gebildet, an dem sich in erster Linie die epigastrischen Gefäße beteiligten, ein eigentliches Caput medusae fehlte.

Kaum 14 Tage nach der Vorstellung begann dann der Tumor mit unheimlicher Schnelligkeit wieder zu wachsen, an dessen erneute Entfernung nun natürlich nicht mehr zu denken war. Sehr bald war der Umfang des Leibes vor der Operation wieder erreicht und der Bauch schlieBlich in eine vollkommene Kugel verwandelt. Unter zunehmendem Kräfteverfall erfolgte am 2. V. 1906 der Exitus letalis.

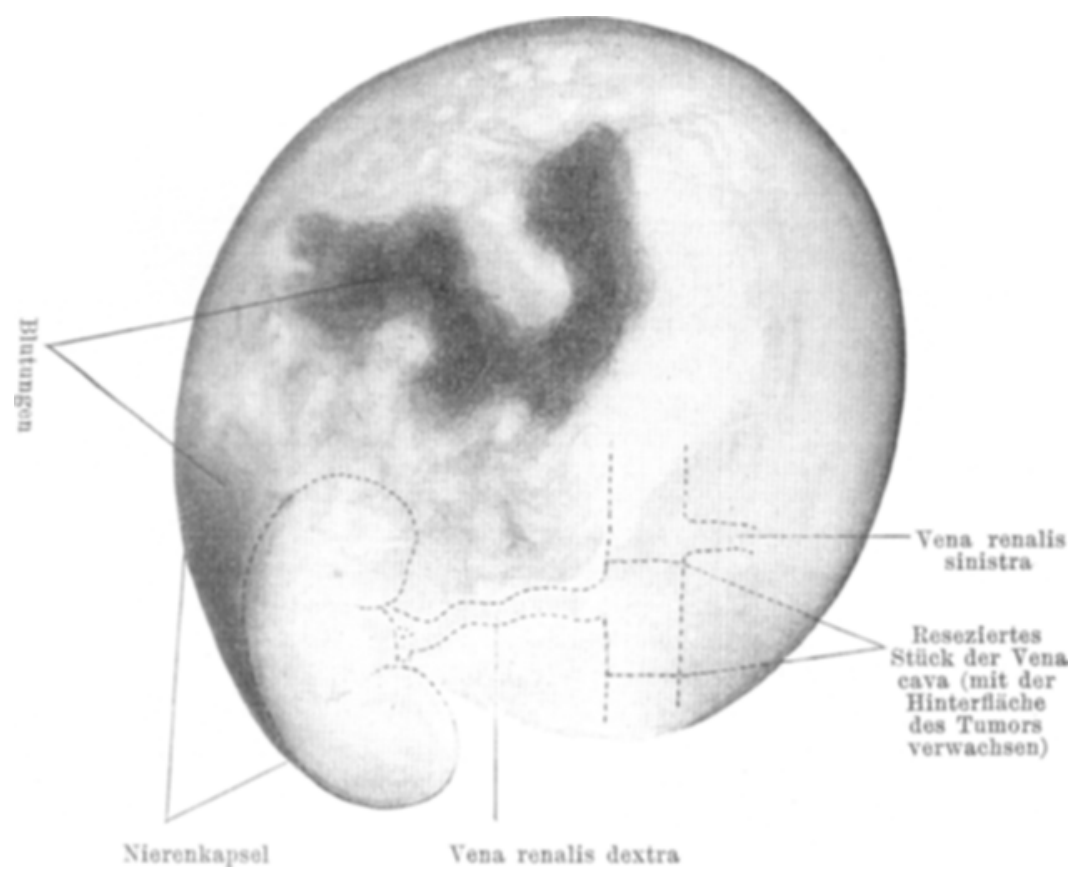

Fig. 1.

Die beiden Punkte, die eill ganz besonderes Interesse bei unserem Fall beanspruchen, die Art des Tumors einerseits und die Tatsache der Cavaresektion andererseits, erfordern, ${ }^{*}$ um Wiederholungen zu vermeiden, eine Zweiteilung des Stoffes. Zunächst sei also über die Natur des Tumors berichtet.

Die beigefügte Slizze mag die Lagebeziehungen des Tumors zu Niere und Kapsel sowie die ungefahre Lage der Reseltionsstelle der Cava veranschaulichen helfen.

Die Untersuchung des Tumors, die zum Teil im hiesigen pathologischen Institut (Prof. Beneke) vorgenommen worden ist, forderte folgende, sehr interessante Verhältnisse zutage. 
Makroskopisch ergab sich zunächst schon unmittelbar nach der Operation, daß die Niere selbst auf den ersten Blick gar nicht an der Geschwulstbildung beteiligt schien; sie sah außerlich im wesentlichen normal aus und lag dem unteren Pol der Geschwulst nur an, oder war zum Teil in ihn hineingeschoben.

In der Länge miBt dic Niere $9 \mathrm{~cm}$, ihre Rinden- und Marksubstanz ist blaß, verschmälert; an den dünnsten Stellen messen beide zusammen nur $8 \mathrm{~mm}$. Das Nierenbecken ist dilatiert, glatt, blap, seine Schleimhaut verdickt. Die Nierenkapsel ist in der oberen Hälfte des Organes durch ein frisches, flaches Hämatom abgehoben; weiter nach aufwärts wird sie derber und dicker und bildet einen über kindskopfgroßen Sack, dessen Wanddicke 1-2 mm hetriggt. Die AuBenwand dieses Sackes ist von Fettgewebe berleckt und ziemlich glatt. Nebennierenteile werden an derselben nicht gefunden. Die Innenflache erscheint etwas gefeldert, der Innenwand einer Harnblase nicht unähnlich. Überall sitzt derselben ein weiches, oft hämorrhagisch infiltriertes grauweibliches Geschwulstgewebe an. Dasselbe ist markweich, sehr zerreißlich und enthält Abschnitte mit sarkomåhnlichem Bau, nämlich GefäBstränge mit grauem Sarkommantel, auBerdem aber eine Anzahl selir kleiner, praller, wasserklarer Cysten. Diese Tumormasse geht direkt in das Hämatom der Kapsel über. Das Nierengewebe ist in der Gegend des oberen Nierenpoles oberfláchlich von dem Tumor ergriffen und sieht wie angenagt aus, doch dringt die Wucherung nirgends tief ins Nierengewebe ein. Die BlutgefảBe des Tumors sind z. T. mit geronnenem Blut gefüllt. Alle Blutungen mit Ausnahme einer Stelle scheinen erst kürzlich entstanden zu sein.

Die rechte Nierenvene ist stark zusammengedrückt, sie ist thrombenfrei und geht spaltförmig in die Vena cava üher, von der ein etwa $3 \mathrm{~cm}$ langes Stück an dem Präparat hängt. Auch dieses Stück ist frei von Thrombose. Die Einmündungsstelle der linken Vena renalis ist an dem resezierten Venenstück nicht mitgetroffen.

Die mikroskopische Untersuchung der' Tumormassen labt an den meisten Stellen deutlich entwickelte Drüsengänge erkennen. Dieselben bilden entweder schmale, an embryonale Tubuli recti erinnernde Gänge oder größere Gruppen engerer und weiterer Kanäle mit einem mehr oder weniger deutlichen, sehr dichtgedrängten Zylinderepithel, welches bisweilen mehrschichtig erscheint. Die Zellen sind annähernd gleich groB, ihr Protoplasma indifferent. Die Iumina der Kanäle sind relativ eng, enthalten vereinzelt kleine, hyaline Zelltrümmer und auch rote Blutkörperchen. Diese Epithelkanäle ruhen vielfach auf einem mäßig zellreichen Stroma, welches in Form schmaler Papillen zwischen den Kanälen aufsteigt, so daß Bilder wie bei einem papillären Adenom entstehen. An anderen Stellen umschließt das Stroma breitere Gruppen der Kanäle und dringt nur spärlich zwischen dieselben oin, bildet aber zwischen den Gruppen eine reichliche Spindelzellwucherung. In diesen Abschnitten finden sich vielfach frische Blutungen.

Endlich gibt es Partien, in welchen das Epithelgewebe einem kleinzelligen Rundzellengewebe aufsitzt, dessen Kerne den Epithelien so dicht ansitzen und derartig gleichen, daf eine Abgrenzung beider Formen streckenweise große Schwierigkeiten hat. Dieses zellreiche, sarkomähnliche Ge- 
webe hebt sich von dem übrigen Stroma scharf ab und zeigt besondere Neigung zu Nekrose und Blutungen. In manchen Partien bildet es isoliert, ohne Drüsen, größere Abschnitte. Diese Teile gleichen dann durchaus einem kleinzelligen Sarkom und zeichnen sich auch durch ihren Reichtum an weiten GefäBen aus.

In einem anderen Bezirk habe ich dann noch in einem vorwiegend spindelzelligen, sehr lockeren Stroma, das aber auch vereinzelt sternförmige Zellen enthält, gröBere oder kleinere Züge von glatten Muskelfasern gefunden, die stellenweise $\mathrm{zu}$ förmlichen Zöpfen oder breiten Bändern anwachsen, die sich in ganz unregelmäBiger Anordnung kreuz und quer durchflechten. Wie in dem von Vogler beschriebenen Fall, den Wilms erwähnt, erinnern die letztgenannten in ihrer breiten Ausdehnung an quergestreifte Muskelfasern, doch fehlt die Querstreifung.

In Übereinstimmung in allen wesentlichen Punkten mit den bisher über diesen Gegenstand in der Literatur niedergelegten Daten ergibt sich also aus unserer Untersuchung, daß es sich um eine in ihrer Erscheinungsweise typische embryonale Mischgeschwulst der Niere handelt.

Nach dem Ausspruch von Wilms, diese Gebilde unbedingt als einen für sich wachsenden "Privatorganismus" aufzufassen, der seine Topographie für sich besitzt, habe ich versucht, durch eine auf die verschiedensten Regionen sich erstreckende Untersuchung über die Gesamtstruktur Aufschluß zu erhalten. Außer den oben genannten habe ich aber keine anderen fremdartigen Bestandteile, wie Knorpel oder gar Knochen, finden können.

Knochen ist übrigens kürzlich zum ersten Male von Hedrén in einem derartigen Tumor gefunden worden und damit gewissermaßen eine theoretische Forderung im Sinne von Wilms erfüllt worden. Das seither stets beobachtete Fehlen von Knochen hat Wilms bekanntlich auf rein mechanische Einflüsse zurückführen wollen, indem nach ihm die zur Knochenbildung notwendigen äußeren Wachstumsbedingungen fehlen; die Mög]ichkeit der Entwicklung von Knochen mußte natürlich nach seiner Hypothese vorliegen. Durch das nunmehr festgestellte Vorkommen von Knochen werden meiner Auffassung nach zugleich die letzten Schwierigkeiten weggeräumt, die sich Wilms noch bei Beantwortung der Frage entgegenstellten, ob primäre Mesenchymkeime in der Tat die Mutterzellen für die Bindegewebsformationen in den Nierenmischtumoren sein können. Hedrén, der hinsichtlich der Pathogenese ausführlich auf die Hypothesen von Wilms und Busse eingeht, neigt trotzdem mehr der Birch-Hirschfeldschen Anschaung zu, wenn er auch die Entscheidung noch von weiteren embryologischen Studien abhängig machen will.

Deutsehe Zeitschrift f. Chirurgie. 88. Bd. 
Solange noch keine sicheren Beweise vorliegen, ob man die Entstehung dieser Tumoren aus Mesenchymzellen der frühesten Fötalperiode oder aus der fertigen Nierenanlage herleiten soll, bleibt es persönliche Ansichtssache, welcher der beiden Anschauungen man sich zuwenden will. Meinem Gefühl nach entspricht am ehesten trotz der vielen entgegengesetzten Ansichten die erste Hypothese und ich stehe auf dem Boden der Wilmsschen Auffassung, wenn ich annehme, daß bei unserem Tumor sich aus einem zunächst noch undifferenzierten embryonalen Keimgewebe Bindesubstanzelemente einerseits und Bildungen epithelialer Natur andererseits entwickelt haben. Aus der Schwierigkeit, in unserem Falle epitheliale oder nichtepitheliale Elemente stellenweise mit Sicherheit zu trennen, ist jedenfalls der Schluß erlaubt, daß die einen aus den anderen entstanden sind. Hier mag man nun annehmen, wie ich es eben andeutete, daß aus einem ursprünglich embryonalen Keimlager, das durch Versprengung aus den mesodermalen Zellen der Urnierengegend als Matrix hervorging, sich Zellanhäufungen bilden, die, von mehr spindeligen Elementen umgeben, allmählich immer mehr und mehr epithelialen Charakter annehmen und sich schließlich zu vollkommenen Drüsenschläuchen heranbilden. Andere und die Mehrzahl haben die gerade umgekehrte Auffassungsweise, wenn sie Birch-Hirschfeld folgen, nach dessen Meinung die Drüsen durch atypische Wucherung zu rundzelligen Elementen werden sollen.

Der erste Anstoß zur Keimverlagerung mag in einem in frühestem Embryonalstadium erworbenen Trauma liegen. Was die Ursache des postembryonal beginmenden unbegrenzten Wachstums sein mag, ob ein erneutes Trauma, ein Anstoß unbekannter Natur von innen heraus, Attraxinwirkung, wir wissen es noch nicht.

Freund hat auf der 78. Naturforscherversammlung geäußert: es läßt sich denken, daß der Vorgang der Weiterentwicklung einer dislocierten oder in der Teilung zurückgebliebenen Blastomere auch im späteren Leben vorkommen kann, d. h. daß es erworbene Embryome gibt. Aber das Wie bleibt vorläufig dunkel. Warum entstehen diese Geschwülste meistenteils im jugendlichen Alter? Es bedarf doch offenbar nur eines ganz geringen Anstoßes, um die enorme Wachstumstendenz solcher Tumoren zum Ausbruch anzuregen, die ganz der embryonalen Wachstumsschnelligkeit entspricht, wie sie im späteren Leben nirgends wieder erreicht wird. Sollte man in dieser ungeheuren Wachstumsenergie nicht auch einen Hinweis 
erblicken können, das Datum der Keimversprengung in die allerfrühzeitigste Periode zu verlegen?

Auf dem anderen Boden bezüglich der Erklärung der Geschwulstgenese stehen neben vielen anderen Busse und Muus, die die Mischgeschwülste aus der fertigen Niere entstehen lassen. Gegenüber ihrer Erklärung führt Wilms an, daß mehrfach kindliche Mischtumoren außerhalb der Nierenkapsel lagen und meint, daß diese Tatsache sich eher für eine Abstammung aus der Urniere, als aus der Niere verwerten läßt. Hingegen muß gerade dieser Grund als hinfällig angesehen werden, seit Imbert auf Serienschnitten durch junge menschliche Embryonen neben der Niere in der Kapselanlage kleine abgesprengte Partikel von Nierenstruktur gefunden hat, welche durch einen feinen Stiel mit der Hauptniere zusammenhängen. Imbert meint, daß vielleicht solche außerhalb des Hauptorganes angelegte Zellhaufen bei weiterer Entwicklung die in Rede stehenden Mischgeschwülste bilden, wobei nach ihm aber immer noch die Entstehung der quergestreiften Muskeln weiterer Aufklärung bedarf. Früher hatte er dieselben durch Metaplasie aus den in der normalen Niere des Embryo sehr reichlichen glatten Muskeln entstehen lassen. Wenngleich man nicht gerne an den Gedanken der Metaplasie herangeht, würde sich vielleicht dafür das Aussehen der Muskelfasern unseres Falles sowie zahlreicher Zwischenstufen aus anderen Fällen verwerten lassen, die äußerlich bis auf die fehlende Querstreifung viel Ähnlichkeit mit quergestreiften Muskelfasern habell.

Busse weist darauf hin, daß im embryonalen Muskelgewebe die Muskelfasern mit zunehmendem Alter an Breite und Länge wachsen und daß dementsprechend auch die Differenzierung des Protoplasmas fortschreitet, mit anderen Worten, auch die Querstreifung deutlicher wird. Es steht also die Breite der Fasern in einem direkten Verhältnis zu dem Alter und der Querstreifung der Fasern. In den embryonalen Geschwülsten besteht nun in der Tat ein solches Verhältnis nicht, sondern gerade, wenn die Fasern breit entwickelt sind, ist oft, wie auch in unserem Fall, von Querstreifung entweder überhaupt nichts zu sehen, oder sie ist nur gerade in ihren ersten Anfängen angedeutet. Wollte man also den Maßstab zur Beurteilung solcher Fasern aus der Entwicklungsgeschichte entlehnen, so würde man bei der Bestimmung des Alters auf Schwierigkeiten stoßen. Busse behauptet nun, daß solche Muskelfasern bei der normalen Muskelentwickelung überhaupt nicht vorkommen und dalis in solchen Geschwülsten die Metaplasie aus 
der glatten Muskelsubstanz in quergestreifte erst sehr spät oder nur äußerst unvollkommen eintritt.

Der Ansicht der Gegner der Wilmsschen Theorie wird am besten Ausdruck verliehen durch die Zusammenfassung, die Jenckel als Schluß einer bemerkenswerten Arbeit über die embryonalen Drüsengeschwülste der Niere gibt:

Es ist nicht nötig, für alle Nierenmischgeschwülste nach der Hypothese von Wilms eine Keimversprengung aus frühester Fötalperiode anzunehmen, da in der Nierenanlage selbst ein Gewebe vorhanden ist, aus welchem sich alle Bestandteile, welche die Mischgeschwulst zusammensetzen, erklären, bezw. ableiten lassen. Das Vorkommen ektodermaler Bestandteile in diesen kompliziert zusammengesetzten Nierentumoren spricht eher gegen als für die Theorie von Wilms, da die Beziehungen zwischen Wolffschem Gang und Ektoderm erst hervortreten zu einer Zeit, wo die Differenzierung des Mesoderms in Myo- und Nephrotom bereits durchgeführt ist. Jedenfalls lassen sich die ektodermalen Bestandteile durch Störungen in dem späteren Stadium der Entwicklung als Produkte der Nierenkeimanlage selbst erklären.

Bezüglich des Ausgangspunktes für unseren Tumor besteht eine Abweichung von dem gewöhnlichen Bilde, insofern ich den Beginn der Wucherung als außerhalb derNiere entstanden annehmen möchte. Er reiht sich damit den sehr seltenen Fällen an, in denen man die Nierenkapsel als Ort des ersten Beginnes des Geschwulstwachstums ansehen kann. Wilms konnte 1899 nur 2 Fälle, als mit Wahrscheinlichkeit in dieser Weise zu deuten, anführen, nämlich die von Brock und Vogler.

L. Bork hat 1901 eine Zusammenstellung von Nierenkapselgeschwülsten gegeben. In der überwiegenden Mehrzahl handelt es sich um Myxolipome und Fibrome. Aber einer der Fälle ließe sich vielleicht in unsere Kategorie mit einbeziehen und zwar der Fall Wagner-Thiersch (Nr. 9 der Tabelle). Hier handelte es sich bei einem 3jähr. Mädchen um ein großes Sarkom, welches von der Nierenkapsel ausgehend die Substanz der Niere fast ganz unberührt gelassen hatte. Leider fehlen nähere Angaben über die Natur des Tumors, so daß man nur aus dem Alter des Kindes und aus dem klinischen Verlauf, der mit dem unsrigen viel Ähnlichkeit aufweist, namentlich auch bezüglich der Verhältnisse der Vena cava, vermutungsweise auf Analogie schließen kann.

In dem Umstand, daß die Niere selbst in ihrer äußeren Form vollkommen erhalten dem unteren Tumorpol anliegt, während die Geschwulst unter Abhebung der Nierenkapsel sich zwischen diese und Niere hereinschiebt, scheint mir doch ein wichtiges Zeichen 
dafür zu liegen, daß der Tumor extrarenal entstanden ist. Daß die Niere am oberen Pol vom Tumor wie angenagt erscheint, indem die Wucherung stellenweise, wenn auch nicht tief, in sie hineindringt, spricht meines Erachtens nicht dagegen, sondern im Gegenteil dafür, denn es scheint zu beweisen, daß das Geschwulstwachstum von außen her kam und sich gegen die Niere richtete. Die letztere beteiligt sich ja erfahrungsgemäß in keiner Weise an dem Wachstum der Geschwulst.

In allen anderen Punkten reiht sich unser Fall durchaus gleichartig dem bisher beobachteten an: das Vorkommen bei einem jugendlichen Individuum, das enorm schnelle Wachstum, Fehlen von Blut im Urin im Gegensatz zu den Hypernephromen der Erwachsenen, das äußerst schnelle Rezidivieren nach der Exstirpation, das alles sind bekannte Erscheinungen. Ich kann an dieser Stelle gleich erwähnen, daß die Sektion keine Metastasen in anderen Organen ergab (die wie gewöhnlich bei Mischtumoren nur relativ selten, etwa in 30 Proz. der Fälle auftreten); auch konnte in der anderen Niere kein Tumor gefunden werden, während doppelseitiges Vorkommen hier verhältnismäßig oft beobachtet wurde.

Ich komme nun nach Besprechung der pathologisch-anatomischen Verhältnisse zum zweiten Teil meiner Betrachtung, die sich mit der Resektion der Vena cava befassen soll. Eine Naht der Vene bietet hentzutage so wenig Ungewöhnliches mehr, daß ich diese Fälle außer acht lassen kann und nur die wirklichen Unterbindungen beziehungsweise Resektionen im Gebiet der Vena cava gelegentlich der Besprechung unseres Falles berücksichtigen will.

Ehe ich auf die Frage nach der Berechtigung zu diesem Eingriff und seine Folgen eingehe, will ich kurz noch einmal erwähnen, daß bei der Operation unseres Falles die Sache so stand, daß die Resektion der $V$. cava sich als notwendig herausstellte, wenn man überhaupt die begonnene Operation zu Ende führen und an eine, wenigstens nach menschlicher Voraussicht, radikale Entfernung des Tumors denken wollte. Der Tumor ging untrennbar in die Venenwand über und in ihr auf, so daß eine einfache Naht der Venenwand nach Ablösung der Geschwulst gar keinen Wert gehabt hätte.

Einzelne Autoren stehen allerdings auf dem Standpunkt, daß die kindlichen Nierentumoren überhaupt ein Noli me tangere für den Chirurgen darstellen. Da in neuerer Zeit aber ab und zu Fälle veröffentlicht wurden, in denen eine embryonale Mischgeschwulst 
der Niere exstirpiert wurde, ohne daß ein Rezidiv auf dem Fuße folgte (allerdings ist die Beobachtungszeit meist keine sehr lange gewesen), ist man, glaube ich, verpflichtet, solche Tumoren doch zu operieren. Auch Israel sieht trotz der besonders großen Recidivfähigkeit dieser Geschwülste des Kindesalters keine Gegenanzeige gegen die Operation.

Der Eingriff selbst gestaltete sich bei uns außerordentlich einfach. Sobald nach Beiseitewälzen des Tumors klar war, daß man die mit ihm verwachsene Vena cava vor sich hatte, war das Handeln ein gegebenes. Die reichliche Venenentwickelung auf der Bauchhaut, sowie das bandartige Zusammengedrücktsein des Gefäßes, das sich auch beim Emporheben des Tumors und dadurch bewirkter Lüftung des Lumens nicht bemerkbar stärker füllte, ließen mit Sicherheit annehmen, daß sich bereits ausreichende Kollateralen gebildet haben mußten, um die Vene ohne Gefahr wegnehmen zu können. Es wurde deshalb schnell, um so mehr als der Zustand des Kindes im Verlaufe der Operation eine rasche Beendigung erheischte, ober- und unterhalb der Verwachsungsstelle je ein Katgutfaden um das Gefäß gelegt, der noch durch eine daneben gelegte Seidenligatur gesichert wurde und nach Abbindung das ganze etwa $3 \mathrm{~cm}$ lange Stück reseziert.

Irgendwelche Schwierigkeiten bei Isolierung des Gefäßes haben sich bei uns nicht herausgestellt. Goldmann gibt z. B. bei seinem Fall an, daß es ihm nur mit großer Mühe gelungen sei, die Vena cava von der Aorta $\mathrm{zu}$ isolieren. Davon haben wir in unserem Fall nichts wahrgenommen. Es gelang überraschend leicht, die Unterbindungsfäden zu legen und nach Durchschneidung peripherund zentralwärts folgte das resezierte Gefäß mit dem herausgewälzten Tumor ohne weiteres.

An welcher Stelle die Unterbindung lag, das zeigte sich erst nach Betrachtung des Präparates, beziehungsweise ergab es zum Teil erst die Sektion. Das Präparat zeigte, daß die rechte Nierenvene in den Bereich der Resektion gefallen war. Sie war stark zusammengedrückt und ging spaltförmig in die V. cava über, war im übrigen thrombenfrei. Über die Lage der linken $V$. renalis konnte bei der Operation keine Sicherheit gewonnen werden. Erst der weitere Verlauf und die Sektion ergaben, daß sie proximal, $d . h$. also herzwärts von dem abgebundenen Gefäßbezirk gelegen war.

Im großen ganzen ist die Tatsache bei Fällen wie in dem unsrigen ohne allzu große Bedeutung, ob die Nierenvene ober- oder unterhalb der Ligatur einmündet. Gesetzt, die Stelle der notwen- 
digen Ligatur läge so ungünstig, daß die Vene der gesunden Niere mit in den Bereich der Unterbindung fiele, so wäre eben das Kind liöchstwahrscheinlich mit und ohne Operation sicher verloren. Eine Wandresektion mit folgender seitlicher Naht dürfte bei so breiter Verwachsung nicht in Betracht kommen.

Der Weitertransport des venüsen Blutes anf dem gewöhnlichen Wege war durch den Druck des wachsenden Tumors doch schon seit einiger Zeit aufgehoben und die außer Funktion gesetzte Cava zwang das venöse Blut anch der linken Niere, sich frühzeitig genug andere Gefäßbezirke als Kollateralbahnen zugänglich zu machen, so daß man eigentlich annehmen kömnte, daß die letzteren ausreichen müßten. Experimente haben aber gezeigt, daß trotzdem in einer Reihe von Fällen der 'Tod infolge schwerer Sclı̈digungen der Niere eintritt. Bei Extremitätengefäßen würden die Kollateralbahnen in jedem Falle ausreichend sein. Goldmann hat zum ersten Male darauf hingewiesen, daß die großen Abdominalgefäßstämme eben eine zweifache Aufgabe zu erfüllen habell, daßs sie auler ihrer Leitungsfunktion auch Ernährungsfunktionen zu übernelımen haben, denen die Kollateralbahnen offenbar nicht in ausreichendem Maße gewachsen sind. Allerdings haben sich dem eben Gesagten andere Autoren entgegengestellt. 'Tuffier hält es z. B. nicht für erwiesen, daß Unterbindung der Tena cava selbst innerhalb des Bereiches des Nierenstieles notwendig die zweite Niere geführdet hätte. Wenigstens hat er experimentell vielfach die Nierenvene abgebnnden, ohne darnach die entsprechende Niere verändert zu finden. Es wären also ernente Experimente anzustellen, um die Bedingungen zu ergründen, unter denen es möglich ist, mit Erhaltung der zweiten Niere die (ava anch oberhalb zu ligieren. ${ }^{\text {) }}$

1) Ein Beispiel für diese Möglichkeit liefert ein von W endel beobachteter Fall von Thrombose der Cava inferior, die nach lumbaler Nephrektomie wegen Epinephroid eingetreten war. Trotz des Verlustes der rechten Niere, und trotzdem die Geschwulstthrombose bis in den rechten Vorhof reichte, konnte der Kranke noch längere Zeit weiter leben. Die linke Niere erwies sich bis zuletzt als suffizient. Die hervortretenden Symptome: häufiges Erbrechen, pralle Ödeme an beiden Beinen, eine Hämaturie, die als Stauungsblutung aufgefaßt wurde, sowie die starke Entwicklung der Unterhautvenen an Oberschenkeln und Bauch ließen die Diagnose schon bei Lebzeiten stellen. Nach dem Tode ließ sich durch Injektion von roter Leimmasse, die von der linken V. femoralis aus vorgenommen wurde, nachweisen, daß sich der Nierenkreislauf vollkommen wieder hergestellt hatte. Durch Vermittlung der V. lumbalis fïllten sich die mächtig erweiterten Kapselvenen und mikroskopische Schnitte zejgten, daß die Injektion bis in die Capillaren linein gelungen war. Der 
Wesentlich anders liegt die Sache natürlich bei der „ligature brusque" der Franzosen, wo die Cava-Unterbindung ohne vorbereitende Stenosierung des Lumens erfolgt. In solchen Fällen ist der Tod die unausbleibliche Folge, sobald die Ligatur oberhalb, d. h. zentralwärts von der Einmündungsstelle der Vena renalis angelegt wird. Purpura schließt aus seinen zahlreichen experimentellen Untersuchungen nach Houzel als erste und Hauptfolgerung: „En aucun cas la vie n'est possible avec la ligature au premier temps de la veine cave inférieure en un point quelconque, depuis l'union de la veine iliaque primitive jusqu'au foie; c'est encore plus difficile au-dessus de la veine rénale."

Dieser Schluß ist heutzutage nicht mehr in seinem vollen Umfang aufrecht $\mathrm{zu}$ erhalten, sondern wie zahlreiche Experimente und die Erfolge am Menschen gezeigt haben, ist man durchaus in jedem Falle berechtigt, die Cava zu resezieren, sobald man sich nur unterhalb der Nierenvene hält.

Goldmann hat in seinen Bemerkungen über die Herstellung des venösen Kollateralkreislaufs die Bedingungen erörtert, unter denen derselbe zustande kommt. Er fand, daß die vis a tergo, welche von der Arterie ausgeht, wohl den intravenösen Druck erhöht und die kollateralen Bahnen der ligierten Venen zu entfalten vermag, daß aber neben dieser Kraft noch andere Umstände mitwirken. An der Hand namentlich der experimentell gewonnenen Beobachtungen von $\mathrm{Bier}$ über die reaktive Hyperämie stellt er folgende Momente als maßgebend für die Herstellung des kollateralen Venenkreislaufs hin:

1. Unversehrte Kollateralbahnen,

2. Normaler Blutdruck,

3. Fehlen aller Znstände, die die normale Fähigkeit der kleinen Gefäße, sich gegen venöses Blut zu wehren, beeinträchtigen oder gar autheben,

4. offene Lymphbahnen.

Auf die nähẹre Erörterung dieser 4 Punkte kann ich mich hier nicht weiter einlassen; ich verweise dazu auf die Bemerkungen, die Goldmann hierüber gemacht hat. Ich will nur hervorheben, daß in unserem Falle alle oben aufgestellten Bedingungen erfüllt

Fall zeigt, wie groß die Anpassungsfähigkeit des Kreislaufs durch Ausbildung von Kollateralen bei solchen Gelegenheiten sein kann, und lehrt namentlich auch die Wichtigkeit der Nierenkapselgefäße, wenn die Verlegung der betreffenden Gefäße langsam und allmählich erfolgt. 
waren und also kein Grund vorhanden war, der sich einer regelrechten Herstellung des Kollateralkreislaufs entgegengestellt hätte. $\mathrm{Ob}$ es in unserem Falle zu einer irgendwie bedeutenderen $\mathrm{Neu}$ bildung von Venen gekommen ist, ist schwer zu beurteilen. In der Regel reichen ja die präformierten Bahnen für die Herstellung des definitiven Kollateralkreislaufs aus. Eine wirkliche Nenbildung oder auch nur exzessive Erweiterung vorhandener Venen findet nach Goldmann nur statt, wenn infolge einer Neubildung neben dem Hauptgefäßstamm auch seine Wurzeln und die perivaskulären Lymphräume ergriffen sind.

Ich befand mich in unserem Fall in der glücklichen Lage, die Gefäße der Leiche nach dem Vorgang von Goldmann injizieren zu können. Ich habe zunächst in der von Lexer ausführlich beschriebenen Weise sämtliches Blut aus der Leiche vermittels Durchspritzen mit warmer Kochsalzlüsung ausgewaschen. Dann wurden, um eine reine Injektion nur des Venensystems zu erhalten, die in das Herz eintretenden Gefäße abgebunden und nun in die rechte V. femoralis eine Quecksilberterpentinölverreibung langsam injiziert.

An der Tatsache, daß sich ein noch besseres Injektionsresultat und ein klareres Röntgenbild (vgl. Fig. 2) leider nicht erreichen ließ, tragen verschiedene Umstände schuld. Einmal liegt dieselbe in dem enormen Tumor selbst, der einen ziemlichen Schatten ergibt. Er ist auch die Ursache, daß nur eine relativ geringe Menge der Injektionsflüssigkeit zur Anwendung kommen konnte. Bei größerer Menge derselben bestand die Gefahr, daß der Druck in den Gefäßen, der wegen des abgebundenen Herzens nicht entlastet werden konnte, zu groß geworden wäre und sich das Quecksilber alsdann in den morschen Tumor ergossen und so überhaupt jede Einsicht genommen hätte. Es wäre ja ein Ausweg gewesen, wenn man den Tumor vor der Injektion herausgenommen hätte, allein ich mußte mir sagen, daß bei den starken Verwachsungen dabei so viele Gefäßlumina eröffnet worden wären, daß die Injektionsmasse die freie Bauchhöhle erfüllt und so wieder ein Röntgenbild illusorisch gemacht hätte. Daher mußte ich mich mit dem gewonnenen Resultat begnügen, das immerhin die Punkte, die von Wichtigkeit sind, erkennen läßt. $\left.{ }^{1}\right)$

Im wesentlichen wird auf dem Röntgenogramm das sehr ausgedehnte peri- und prävertebrale Venennetz, das meiner Ansicht nach ganz zweifellose Erweiterung der Venenlumina erkennen läßt,

1) Zur Reproduktion wurden von Herrn Maler Braune die injizierten Gefäßstämme noch übermalt. 


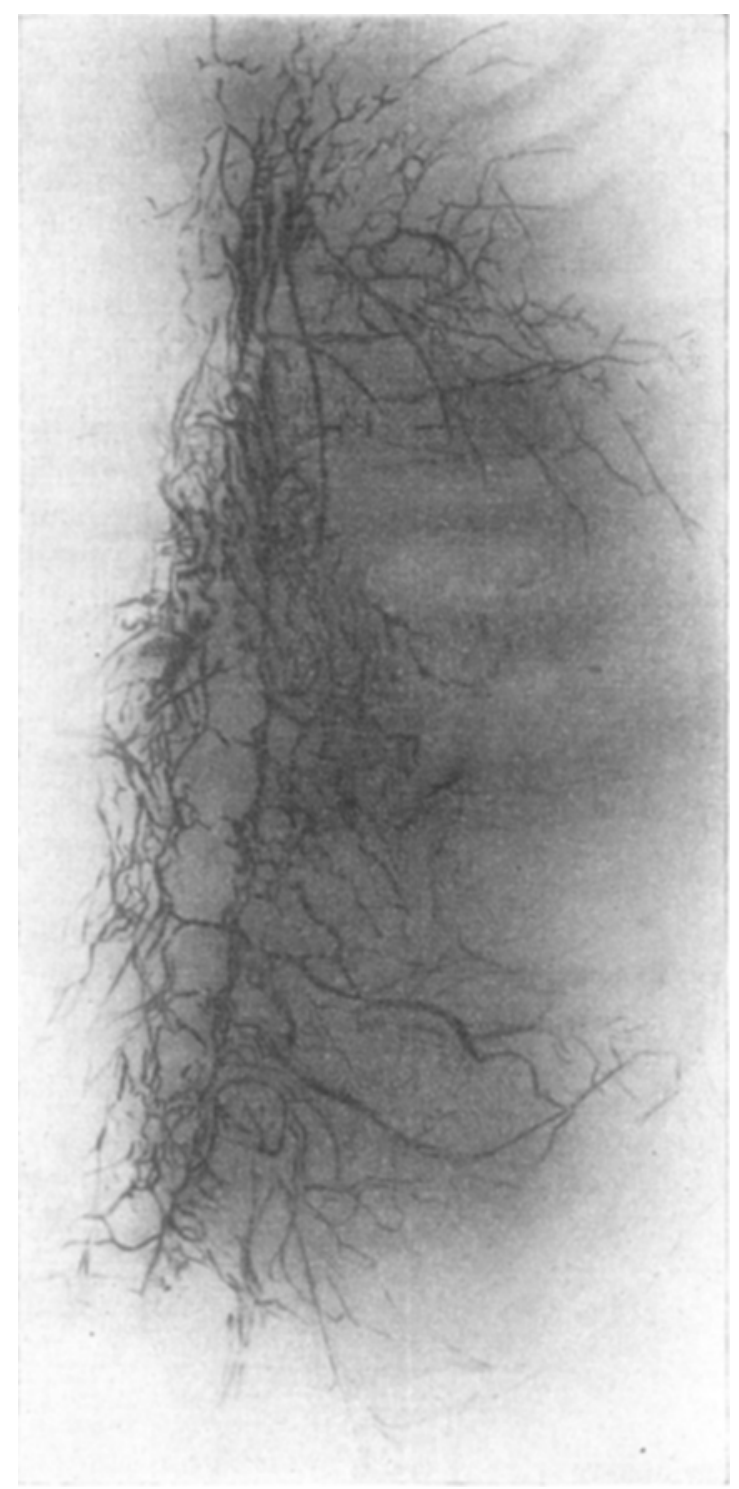

Fig. 2.

sichtbar. Ob sich auch wirklich neugebildete Gefäße darunter befinden, diese Frage ist natürlich schwer zu beantworten. Es macht aber doch fast den Eindruck, als ob in dem Gewirre von venösen 
Gefäßen auch Neubildung Platz gegriffen hätte. Deutlich tritt auf der rechten Seite der Figur die Vena azygos mit im oberen Teil des Bildes aus ihr hervorgehenden Intercostalvenen hervor; sie scheint nicht erweitert zu sein. Weniger deutlich wird die Vena hemiazygos aus dem Gewirre der Venenverzweigungen herausdifferenziert. Eine beträchtliche Erweiterung scheinen dagegen die Vv. lumbales erfahren zu haben, die man besonders auf der Tumorseite als stark entwickelte Stämme verlaufen sieht. Man sieht ferner die Gefäße, die von der linken gesunden Niere herkommen.

Schließlich ist, wenn auch nur unvollkommen, die Entwicklung der Anastomose zwischen Mammariae intt. und epigastrischen Gefäßen angedeutet. Diese Gefäße liegen infolge der kugelförmigen Wölbung des Bauches durch den Tumor natürlich weit ab von der Platte und sind deshalb nicht so klar wie die der Platte zunächst liegenden prävertebralen Venen. Aber man erkennt doch mit hinreichender Deutlichkeit, wie die Mammariae intt. in nach außen leicht konvexem Bogen herunterkommen und ihnen entgegen sich die epigastrischen Gefäße in ebenfalls nach außen konvexem Bogen hinaufziehen. An dem Körper des Kindes war das eben geschilderte Verhalten natürlich weit deutlicher festzustellen, als auf der Röntgenplatte.

Durch unser Röntgenbild erfahren die experimentell gewonnenen Befunde anderer Autoren ihre Bestätigung und man sieht, daß auch beim Menschen ein ganz übereinstimmendes Resultat nach operativer Resektion der $\mathrm{V}$. cava inferior sich einstellt.

Nach Poirier umfassen die Kollateralbahnen die Vena azygos, Vv. lumbales und sacrales, diaphragmaticae, vertebrales, renales und deren Fettkapseläste, die Venen der vorderen Banchwand und schließlich die Vena portarum mittels ihrer zahlreichen Anastomosen mit der Cava. Über die letzteren machte Sinclair-White auf der 7t. Jahresversammlung der Brit. med. Assoz. zu Toronto (19106) eine Bemerkung gelegentlich der Besprechung von Störungen des Pfortaderkreislaufs, indem er auf das sog. "Sappeysche akzessorische Pfortadersystem" hinwies; damit sind die Venen gemeint, die im Lig. falciforme und rotundum der Leber verlaufen. Außer diesem Weg führt er noch 3 andere Gefüßbezirke an, die Verbindungen zwischen dem Pfortadersystem und dem übrigen Gefäßsystem bilden, nämlich Anastomosen zwischen den Venen des Magens und der Speiseröhre, Anastomosen zwischen den 3 Hämorrhoidalvenen und schließlich das Retziussche Venensystem, das retroperitoneal hinter Pankreas, Duodenum und Colon transversum verläuft. 
Unser Röntgenbild ergibt nicht, daß der eine oder andere von diesen Wegen in unserem Fall ausgedehnter mit benutzt worden wäre. Allenfalls ließe sich das nur von dem Hämorrhoidalgeflecht sagen, das ja durch den Plexus hypogastricus eine direkte Terbindung zwischen Pfortadersystem und unterer Hohlvene herstellt. Ganz ähnliche Bilder wie wir hat Goldmann erhalten, der Föten, bei denen die V. cava reseziert war, von der Vena femoralis aus eine Wismut-Öl-Emulsion injizierte. Besonders deutlich fand auch er die prä- und intravertebralen Kollateralbahnen ausgebildet. Diese scheinen bei der Verödung der Cava in erster Linie benutzt zu werden, wie dies bereits Poirier zum Ausdruck bringt, indem er sagt: Suivant le siège de l'oblitération c'est telle ou telle de ces voies, qui est utilisée de préférence. Mais d'une manière générale ce sont les veines rachidiennes, soit extérieures, azygos et lombaires, soit les plexus intrarachidiens, qui sont la principale voie de retour."

Die 6 bisher bekannt gewordenen Fälle von Cava-Unterbindung beziehungsweise Resektion seien hier kurz zusammengestellt:

1. Küster. Lumbale Nephrektomie wegen infiltrierenden Carcinoms. Resektion der V. cava. Tod infolge Anurie 26 Std. post op.

2. Bottini. Exstirpation eines prävertebralen Lymphosarkoms. Einriß der Vena cava; Resektion; vorübergehendes Ödem. Heilung.

3. Houzel. Nephrektomie wegen Pyonephrose, Einriß der Vena cava, Ligatur; vorübergehendes Ödem. Dauerheilung.

4. Heresco. Nephrektomie wegen Steinpyonephrose auf transperitonealem Wege. Verletzung der Vena cava, Ligatur; keinerlei Störungen. Heilung.

5. Hartmann. Transperitoneale Nephrektomie wegen Pyonephrose. Einriß der Vena cava, Ligatur. Heilung ohne Zwischenfälle.

6. Goldmann. Nephrektomie wegen Pyonephrose; Einris der Vena cava; Ligatur; Exitus.

Diesen Fällen reiht sich an als

7. Lexer. Exstirpation einer embryonalen Nierengeschwulst. Verwachsung mit der Vena cava. Resektion. Heilung ohne Kreislaufsstörungen. Exitus 1/2 Jahr später wegen Rezidiv.

Aus allen diesen Fällen geht die vielfältig schon vorher durch Experimente festgestellte Tatsache hervor, daß man durchaus berechtigt ist, die Vena cava unterhalb des Zuflusses der Venae renales unbedenklich zi unterbinden bezw. zu resezieren.

Namentlich haben Gosset und Lecène nach ihren Versuchen das in dem Satz zum Ausdruck gebracht, daß die Unterbindung der 
Cava an eben genannter Stelle in keinem Falle von irgendeiner pathologischen Erscheinung gefolgt ist: es tritt meist nicht einmal Ödem der unteren Extremitäten auf.

Bedenklich wird die Ligatur erst, wenn man sie oberhalb der Einmündungsstelle der Nierengefäße vornimmt; dann treten allerdings trotz ausreichend erscheinender Anastomosen schwere Schädigungen der Nieren ein, die in kurzer Zeit den Tod zur Folge haben können. Ein abschließendes Urteil ist indes zurzeit über diesen Punkt noch nicht zu fällen, da Tuffier z. B., wie ich vorher erwähnte, anderer Ansicht ist.

Welchen Weg man bei Verletzungen der Cava wählen soll, ob seitliche Naht oder Ligatur, bezw. Resektion, das scheint mir in allgemeinen ziemlich gleichgültig zu sein. Heresco tritt allerdings mehr für die Ligatur ein. Er meint, sie sei bei Verletzungen der Vena cava gelegentlich von Nephrektomien vorzuziehen, namentlich wenn es sich um Neubildungen der Niere mit geschwellten Drïsen handelt. Ihm ist es vorgekommen, daß die geschwellten Driisen eine Embolie der Vene veranlassen kömmen, die durch Ligatur zu vermeiden ist.

Den gleichen Standpunkt bezüglich der Naht scheint Lejars einzunehmen. In seinem Bericht über die Ligatur der großen Venenstämme auf dem französ. Chirurgenkongreß 1906 führt er an, daß die Ligatur der Cava inferior, solange sie unterhalb der Nierenveneneinmünduug gelegen bleibt, nicht die ehedem so gefürchteten Zufälle veranlaßt und in der Praxis das beste Verfahren zur venösen Blutstillung bildet. Hingegen bewährt sich die Naht, wie er meint, so verlockend sie auch scheinen mag, nur bei Verletzungen der Vena portarum und der Cava oberhalb des Nierenveneneintritts, wo der plötzliche Stillstand der Zirkulation tödlich wirken würde.

Jedenfalls wird man bei zielbewußtem Vorgehen gelegentlich der Exstirpation großer Tumoren von vornherein nur eine Ligatur oder Resektion ausführen können; eine seitliche Naht wird in den seltensten Fällen möglich sein. Inwieweit die Ausbildung der Gefäßchirurgie imstande sein wird, hier erfolgreich in Tätigkeit zu treten, muß die Zukunft lehren. Im allgemeinen wird der meist elende Zustand der kleinen Patienten eine langdauernde Operation kaum gestatten. 
126 VI. Dratdr, Über Cava-Resekt. in einem Fall von Mischgeschwulst usw.

\section{Literaturverzeichnis.}

1. Wilms, Die Misehgeschwülste. I. Abteil.: Die Mischgeschwülste der Niere.

Lejpzig 1899.

2. Hedrén, Zieglers Beiträge 1906, Bd. IV, Heft 1.

3. Busse, Virchows Archiv, Bd. 157, S. 346.

4. Muus, Virchows Archiv, Bd. 155, S. 401.

5. Imbert, L., Les nouvelles idées sur la pathogénie des tumeurs malignes du rein. Montpellier méd. 1900, S. 462.

6. Derselbe, Sur deux cas de tumeur mixte du rein. Montpellier méd. 1901, Nr. 17.

7. Jenckel, A., Deutsche Zeitschr. f. Chir., Bd, 60, S. 500.

8. Bork, L., Arehiv f. klin. Chir.. Bd. 63, S. 928.

9. Wagner, Deutsche Zeitschr, f. Chir., Bd. 24, S. 505.

10. Israel, Chirurgische Klinik der Nierenkrankheiten. Berlin 1901.

11. Goldmann, Beiträge zur klin. Chir., Bd. 47, S. 162.

12. Houzel, Bull. et mém. de la soc. de chir. 1902, Nr. 18.

13. Derselbe, Revue de Chir., Bd. 27, 5. 285 .

14. Derselbe, Archives provinciales de Chir. Sept. 1905, No. 9.

15. Bier, Virchows Archiv, Bd. 147, S. 256.

16. Lexer, Kuliga und Türk, Untersuchungen über Knochenarterien. Berlin 1904.

17. Poirier, Anatomie humaine, Bd. II, 2, S. 990.

18. Sinclair-White, Ref. Münchner med. Wochensehr. 1906, Nr. 42.

19. Küster, Deutsche Chirurgie, Lief. 52b. Stuttgart 1896-1902, S. 614.

20. Bottini-Houzel, Revue de Chir. 1903, Bd. 27, S. 456.

21. Heresco, Bull. et mém. de la soc. de chir. 1902, No. 40.

22. Hartmann, Bull, et mém. de la soc. de chir. 1904, No. 2.

23. Gosset et Lecène, Ref. bei Goldmann, Beiträge zur klin. Chir., Bd. 47, S. 169.

24. Lejars, Ref. Münchner med. Wochenschr. 1906, S. 2275.

25. Wendel, Verhandl. der Deutschen Gesellsch. f. Chir. 1904, S. 288. 